\title{
Validación de una escala de bullying en adolescentes de instituciones educativas de Medellín, Colombia
}

Luis Felipe Higuita-Gutiérrez

orcid.org/0000-0003-1361-3124

Universidad de Antioquia (Colombia)

hgfelipe87@hotmail.com

\section{Jaiberth Antonio Cardona-Arias}

orcid.org/0000-0002-7101-929x

Universidad de Antioquia (Colombia)

Universidad Cooperativa de Colombia

(Colombia)

jaiberthcardona@gmail.com

\section{Resumen}

El clima escolar y el bullying son fenómenos multicausales cada vez más importantes en el ámbito escolar. En contextos escolares norteamericanos, se han diseñado instrumentos para tratar de valorarlo; no obstante, en Colombia, estos son exiguos. Por lo anterior, se diseñó este estudio con el objetivo de evaluar las propiedades psicométricas del cuestionario de clima escolar y seguridad, en adolescentes escolarizados en instituciones públicas de la ciudad de Medellín, Colombia. La escala se aplicó a 3460 estudiantes seleccionados a partir de un muestreo probabilístico bietápico. Se calcularon frecuencias relativas y absolutas. Se evaluaron las propiedades psicométricas de consistencia interna, fiabilidad, poder discriminante, validez de contenido, predictiva y de constructo. Se concluye que la escala de clima escolar y seguridad en la escuela es un cuestionario corto, de rápida administración, fiable, válido y con excelentes propiedades psicométricas en población adolescente de Medellin para monitorear el clima escolar y el bullying.

\section{Palabras clave}

Escalas de medición; violencia escolar; clima escolar; buylling; Medellín-Colombia (Fuente: Tesauro de la Unesco).

Recepción: 27-02-2016 | Envío a pares: 02-04-2016 | Aceptación por pares: 11-10-2016 | Aprobación: 27-10-2016 


\title{
Validation of a Bullying Scale Applied to Adolescents at Schools in Medellin, Colombia
}

\begin{abstract}
The school climate and bullying are increasingly important multicausal phenomena in the school environment. Instruments have been designed in North American school contexts to try to assess it. However, these are meager in Colombia. For that reason, this study was designed to evaluate the psychometric properties of the school-climate and safety questionnaire for adolescents enrolled in public schools in the city of Medellin (Colombia). The scale was applied to 3,460 students who were selected through a process of two-stage probabilistic sampling. Relative and absolute frequencies were calculated. The psychometric properties of internal consistency, reliability, discriminant power, content validity, predictive and construct were evaluated. It was concluded the school-climate and safety-in-school scale is a short, fast, reliable, valid questionnaire with excellent psychometric properties to monitor the adolescent population in Medellin with respect to the school climate and bullying.
\end{abstract}

Keywords

Measurement scales; school violence; school climate; bullying; Medellin - Colombia (Source: Unesco Thesaurus). 


\section{Validação de uma escala de bullying em adolescentes de instituições educativas de Medellín, Colômbia}

\section{Resumo}

O ambiente escolar e o bullying são fenômenos multicausais cada vez mais importantes no âmbito escolar. Em contextos escolares norte-americanos, foram desenhados instrumentos para tentar valorá-lo; contudo, na Colômbia, estes são exíguos. Por isso, desenhou-se este estudo com o objetivo de avaliar as propriedades psicométricas do questionário de ambiente escolar e segurança em adolescentes escolarizados em instituições públicas de Medellín, Colômbia. A escala foi aplicada a 3460 estudantes selecionados a partir de uma amostra probabilistica bietápica. Calcularamse frequências relativas e absolutas. Avaliaram-se as propriedades psicométricas de consistência interna, confiabilidade, poder discriminante, validade de conteúdo, preditiva e de construto. Conclui-se que a escala de ambiente escolar e segurança na escola é um questionário curto, de rápida administração, confiável, válido e com excelentes propriedades psicométricas em população adolescente de Medellín para monitorar o ambiente escolar e o bullying.

\section{Palavras-chave}

Ambiente escolar; bullying; escalas de medição; Medellín-Colômbia; violência escolar (Fonte: Tesauro da Unesco). 


\section{Introducción}

El clima escolar y el bullying son fenómenos multicausales, cada vez más importantes en el ámbito escolar debido a su relación con la percepción de bienestar, el desarrollo de objetivos sociales, el rendimiento académico, la calidad de la educación, la autoestima de los agredidos y la generación de sentimientos negativos que perduran hasta la edad adulta (Sánchez, Pizza y Rojas, 2009; Ortega et al., 2012). Actualmente, se incluyen entre los fenómenos más reconocidos por la comunidad educativa, por lo que han sido objeto de estudio de disciplinas como la sociología, la psicología, la salud pública y la pedagogía (Prado, Ramírez y Ortiz, 2010). En coherencia con ello, las investigaciones en esta temática se han realizado en múltiples países, incluidos Turquía (Turkemen et al., 2013), Estados Unidos ( Nansel et al., 2001), Italia, España, Inglaterra (Ortega et al., 2012), Argentina, Brasil, México y Colombia (Eljach, 2011).

La cantidad de las investigaciones da cuenta de la importancia de la problemática y, en este sentido, un estudio en 11 países europeos describe prevalencias de bullying que va desde el 10,5\% en Hungría hasta el 29,6\% en el Reino Unido; otros países afectados con este fenómeno incluyen Austria, Países Bajos, España, Suiza, Francia y Grecia (Analitis et al., 2009). Un informe realizado por el United $\mathrm{Na}$ tions Children's Fund (Unicef) sobre violencia escolar en América Latina y el Caribe (Eljach, 2011) señala que la frecuencia de violencia escolar, intimidación o bullying se presenta en el $50 \%$ (Flores, 2009), el $66,1 \%$ (D’Angelo y Fernández 2010) y el 70\% de los estudiantes (Fischer, 2010).

Las múltiples investigaciones y los diversos enfoques adoptados para definir y comprender el clima escolar y la violencia en la escuela, denotan que no existe consenso en la definición de estos conceptos, por lo que abundan las metodologías que pretenden evaluar el impacto emocional, la incidencia, la prevalencia y la gravedad de estos fenómenos, así como las narrativas y los contextos en los que estos se presentan (Rodríguez-Jiménez y Martínez-Rojas, 2009). Entre los múltiples instrumentos diseñados con este objetivo se encuentran los que aplican técnicas de observación, entrevistas (Heinsohn, Chaux y Molano, 2010), grupos de discusión (Síndic de Greuges de Catalunya, 2006) y cuestionarios o escalas (Torregrosa et al., 2011).

Algunos investigadores han sugerido que es preferible la elección de cuestionarios o escalas porque permite evaluar aspectos cognitivos y emocionales difíciles de ser evaluados por observadores externos, obtener una gran cantidad de información con una inversión mínima de tiempo y generar puntuaciones fáciles de interpretar y con garantías psicométricas (Torregrosa et al., 2011). Entre las escalas diseñadas con este propósito, se encuentra la escala de conducta violenta, el cuestionario de agresión reactiva-proactiva, el cuestionario de maltratador/víctima de Olweus, la escala de maltrato de Gatehouse, el Cuestionario de relaciones entre pares, la Escala de burlas para niños-adolescentes (Rodríguez-Jiménez y Martínez-Rojas, 2009), y el Cuestionario de exposición a la violencia (Orue y Calvete, 2010). En Colombia, se han utilizado instrumentos como el Cuestionario de clima social escolar (Prado et al., 2010), el Cuestionario Paredes Lega y Vernon (Uribe, Orcasita y Gómez, 2012; Paredes et al., 2011), el Cuestionario de López et al. (2013), la Escala de Cisneros (Cassiani-Miranda, Gómez-Alhach, Cubides-Munévar y Hernández-Carrillo, 2014), y el California School Climate and Safety Survey (CSCSS) (Sánchez et al., 2009).

En los instrumentos descritos, predominan los que se inspiran en la investigación sobre el bullying, los cuales son muy exhaustivos para definir los papeles que desempeña un estudiante (agresor, víctima u observador) y los tipos de violencia que se han manifestado; no obstante, no incluyen 
el clima escolar que se ha descrito como factor predictor de la agresión entre escolar y así obvian la perspectiva de las relaciones interpersonales de la comunidad académica (Infante et al., 2003; López, Bilbao y Rodríguez, 2012).

En este sentido, se destaca el California School Climate and Safety Survey (CSCSS), un instrumento de 15 ítems desarrollado por Rebelez y Furlong (2013), debido a que en su contenido se encuentran dominios que trascienden el análisis de la violencia o las agresiones en las instituciones educativas, y da cuenta de una manera integral de aspectos relacionados con la convivencia escolar entre estudiantes y profesores, el clima escolar, los conflictos y la victimización. Este instrumento evalúa las percepciones de los estudiantes sobre cuatro dominios: 1. Conductas inseguras, 2. Clima escolar, 3. Condiciones de inseguridad y 4 . Bullying.

En el dominio de conductas inseguras, se pregunta a los estudiantes por la frecuencia en la que fueron testigos de actividades peligrosas en la escuela, tales como peleas, robos, intimidación o amenazas y porte de armas. En el clima escolar, se evalúan las percepciones de los estudiantes sobre las relaciones con los profesores, la percepción que tienen de ellos y algunas políticas de la escuela. Las condiciones de inseguridad trascienden lo que ocurre en la institución al incluir aspectos del contexto cultural y del barrio en el que esta se encuentra, con ítems como el grado en el que la violencia en la comunidad afecta la escuela, la actividad de jóvenes pandilleros y la medida en la que la comunidad académica se ocupa de la seguridad. El bullying indaga sobre la frecuencia en la que el estudiante ha sido agarrado o empujado, golpeado, le han destruido sus pertenencias intencionalmente y lo han amenazado o humillado (Rebelez y Furlong, 2013). En adición a la perspectiva integral de la que parte este instrumento, los estudios previos han descrito las buenas propiedades psicométricas en cada uno de sus dominios, particularmente, la consistencia in terna en las conductas inseguras (a de Cronbach $=$
0,79 a 0,87), el clima escolar (a de Cronbach $=0,78$ to 0,89 ), las condiciones de inseguridad (a de Cronbach $=0,77$ a 0,84) y la victimización (a de Cronbach $=$ o,65 a 0,84) (Rebelez y Furlong, 2013).

El CSCSS ha sido adaptado a población hispana (Infante et al., 2003), donde ha demostrado su utilidad por ser una escala breve, fácil de aplicar, que permite monitorear periódicamente el clima escolar, evaluar las necesidades de los agentes educativos, identificar estudiantes con riesgo de ser víctimas o con historia de maltrato y evaluar la efectividad de las intervenciones realizadas sobre esta problemática (Rebelez y Furlong, 2013). Pese a las ventajas descritas, las investigaciones realizadas en Colombia con este instrumento son exiguas; entre los pocos estudios realizados se encuentra el de Sánchez et al. (2009) y Rodríguez-Jiménez et al. (2009), que, aunque dan cuenta de las bondades de este instrumento, no describen las propiedades psicométricas de la escala en la población colombiana. En este sentido, las investigaciones sobre psicometría y validación de escalas refieren que el desempeño de los instrumentos varía según el perfil de la población de estudio y el constructo que se quiere medir (Fidalgo y Piñuel, 2004), lo cual implica que en cada contexto en el que se aplique un instrumento se debe evaluar el desempeño en términos de validez y confiabilidad.

Esta situación es relevante en cuanto las investigaciones recientes en Colombia han descrito prevalencias de bullying, agresión o maltrato del 20,3\% (Cassiani-Miranda et al., 2014), el 24,7\% (Paredes et al., 2011) y el 47,5\% (López de Mesa-Melo et al., 2013), todas ellas con instrumentos distintos, extensos y con limitaciones en las propiedades psicométricas, lo que impide la comparación de los resultados. De la misma manera, los estudios realizados en Medellín indican que el 68,3\% de los rectores manifiestan que en su institución se han presentado casos de matoneo o bullying (Personería de Medellín, 2013); sin embargo, la disponibilidad de instrumentos que evalúen de manera integral el clima escolar, el 
bullying y la seguridad en la escuela, desde la perspectiva de los estudiantes, son exiguos y además presentan garantías psicométricas casi nulas.

Lo expuesto refleja la necesidad de validar una escala como CSCSS, en cuanto las escuelas dispondrían de un instrumento corto y fácil de aplicar para realizar seguimiento periódico a la situación de cada institución, generar información para que se oriente la participación activa del personal escolar evaluar los progresos realizados hacia los objetivos deseados de seguridad en la escuela y proporcionar información sobre cuándo se deben activar otros servicios de apoyo psicosocial. Aunado a lo anterior un sistema de evaluación periódica sobre los niveles de violencia escolar y la percepción de seguridad en la escuela permitiría que las autoridades encargadas de tomar decisiones identificasen las áreas problemáticas y orientarán la implementación de medidas de prevención o intervención tanto individuales como colectivas (Rebelez y Furlong, 2013).

Teniendo en cuenta lo anterior, se diseñó este estudio con el objetivo de evaluar las propiedades psicométricas del cuestionario de clima escolar y seguridad en la escuela de California, en adolescentes escolarizados en instituciones públicas de la ciudad de Medellín.

\section{Materiales y métodos}

El estudio se realizó a partir de la evaluación de escalas. La escala se aplicó en una muestra representativa de estudiantes de instituciones de educación pública de Medellín. Se seleccionaron 3460 estudiantes de una población de 200.000, con una confianza del $95 \%$, percepción de inseguridad en la escuela del 50\%, precisión de muestreo del 2\% y corrección de muestreo del $15 \%$. Se realizó un muestreo probabilístico bietápico, en la primera etapa un muestreo estratificado con asignación proporcional, que tomó como estratos las siete zonas en que se divide la ciudad; y en la segunda etapa un muestreo aleatorio simple, ya que en cada zona se selecciona- ron de forma aleatoria las instituciones educativas participantes. En las instituciones seleccionadas, se encuestó la totalidad de estudiantes de los grados décimo y undécimo. Cabe aclarar que, en la zona que agrupa los corregimientos, solo se debería muestrear una institución educativa según el muestreo estratificado; sin embargo, a la muestra final se sumaron los estudiantes de tres corregimientos para representar toda el área rural.

Los criterios de inclusión fueron: adolescentes de los grados 10 y 11, habitantes del área urbana y rural, de ambos sexos y de cualquier estrato social. Los criterios de exclusión fueron: estudiantes con alteraciones mentales que pudiesen generar sesgo de memoria, quienes estuvieran bajo el efecto de drogas o alcohol y quienes rechazaron la participación; estos criterios fueron aplicados por psicólogos o profesores de las instituciones educativas.

Recolección de la información: el proyecto fue presentado a los rectores o coordinadores de las instituciones educativas para la realimentación en relación con el trabajo de campo, luego se expuso a los estudiantes para incentivar su participación. Una vez resueltas las dudas de los estudiantes y aplicados los criterios de inclusión y exclusión, se diligenciaron las encuestas. La encuesta contenía información demográfica y el instrumento CSCSS. Antes de esto, se controlaron los sesgos de información inherentes al encuestado, el encuestador y el digitador, y se realizó una prueba piloto con el 10\% de los estudiantes residentes del área rural y de dos zonas del área urbana; en esta prueba, no se hicieron cambios al instrumento, por lo que los datos fueron incluidos en la muestra final.

Análisis de la información: la descripción de los ítems del instrumento se hizo mediante el cálculo de frecuencias relativas y absolutas. La evaluación psicométrica se realizó con los criterios de consistencia interna, fiabilidad, poder discriminante, validez de contenido, validez predictiva y validez de constructo. 
En la consistencia interna, se evaluó el grado de interrelación de los ítems y se verificó que los items que miden cada dominio del CSCSS presentaran homogeneidad entre ellos. Para esto, se calcularon correlaciones de Spearman entre los ítems con su dominio y se consideraron favorables los valores superiores a 0,4; además, se calculó el poder discriminante, que se tomó como exitoso cuando el coeficiente de Spearman fue mayor en las correlaciones ítem-dominio al cual pertenece, en comparación con las correlaciones ítem-dominio al cual no pertenece. La fiabilidad se realizó para dar cuenta del grado de correlación interelementos de los ítems de la escala, que se evaluó a través del coeficiente alfa de Cronbach, y se consideraron satisfactorios valores superiores a 0,7 .

La validez hace referencia a la capacidad del instrumento para medir el constructo para el cual ha sido diseñado. En este caso, se realizó validez de contenido, predictiva y de constructo. Para la validez de contenido, se llevó a cabo análisis factorial exploratorio de componentes principales o cálculo de coeficiente lambda y gráfico de componentes con rotación de varimax; la validez predictiva se calculó con el porcentaje de la varianza del CSCSS explicada por los cuatro dominios de la escala; y la validez de constructo se evaluó con la capacidad del instrumento para diferenciar el perfil de CSCSS en grupos teóricamente diferentes, en este caso hombres frente a mujeres y adolescentes de las diferentes zonas de la ciudad a partir de la prueba chi-cuadrado de Pearson y la prueba Z para la comparación de la subescala de bullying según el sexo. Se consideraron diferencias significativas valores $p<0,05$. Todos los análisis se realizaron en el software SPSS $21^{\circledR}$.

Aspectos éticos. Se respetaron los principios de privacidad, confidencialidad y los demás suscritos en la Resolución 8430 de 1993 del Ministerio de Salud de Colombia y en la Declaración de Helsinki. Este manuscrito se enmarca en la investigación "Bullying en adolescentes escolarizados en instituciones educativas públicas de Medellín", avalada por el Comité de Bioética de la Universidad Cooperativa de Colombia, informe de aprobación 0800-002.

\section{Resultados}

Se encuestaron 3460 adolescentes con una edad promedio de 16 años (rango intercuartil 15 a 16); el $52 \%$ $(n=1815)$ de grado 10; el 60\% $(n=2083)$ mujeres; el 27\% $(n=953)$ de la zona centrooriental; el 17,3\% $(n=598)$ de corregimientos; el 17\% ( $n=593)$ de la zona noroccidental; el 13,6\% ( $n=472)$ de la zona suroriental, y en proporciones similares las zonas suroccidental $(n=344)$, centrooccidental $(n=276)$ y nororiental $(n=224)$.

En los cuatro dominios que evalúan percepción de clima escolar y seguridad en la escuela, se encontró que, en las conductas inseguras, el 21,5\% ( $n=744$ ) de los estudiantes percibe que son entre moderas y altas; en este dominio, los ítems que evalúan la frecuencia de estudiantes que roban fue el $31,3 \%(n=1082)$ y de estudiantes que pelean $28,9 \%$ $(n=1000)$. Sumado a esto, llama la atención que el $4,9 \%(n=169)$ refiere que la frecuencia con la que observa que los estudiantes llevan algún tipo de arma a la escuela es alta (tabla 1).

En el dominio de clima escolar, se incluyen ítems que evalúan las relaciones de los estudiantes con los maestros de la institución, el resultado global indica que el $6,32 \%(n=232)$ de los estudiantes considera que su clima escolar es malo; en los ítems del dominio, se observa que el $11 \%(n=381)$ consideran que sus maestros son injustos y el $7,5 \%(n=260)$ irrespetuosos. En cuanto a las condiciones de inseguridad, el $27,9 \%(n=965)$ perciben algún grado de inseguridad en su escuela. Se destaca que el 23,9\% ( $n=826)$ de los estudiantes consideran que su escuela está siendo afectada por la violencia en la comunidad; de la misma manera, el 6,1\% $(n=211)$ consideran que la presencia y actividad de jóvenes pandilleros en su institución es muy frecuente. En el dominio de victimización o bullying se encontró que el 18,3\% de los adolescentes dicen haber sido víctima de alguna forma de bullying en el último mes; el 8,9\% han sido 
ISSN 0123-1294 | e-ISSN 2027-5358 | Educ.Educ. Vol. 20. No.1 | Enero-abril de 2017 | pp. 9-23.

Universidad de La Sabana | Facultad de Educación

Tabla 1. Descripción de la escala de clima escolar, conductas inseguras y bullying

\begin{tabular}{|c|c|c|c|}
\hline Conductas inseguras & Baja & Moderada & Alta \\
\hline Estudiantes que pelean & 71,1 & 19,7 & 9,2 \\
\hline Estudiantes que roban & 68,7 & 17,2 & 14,1 \\
\hline Estudiantes que amenazan & 80,0 & 12,5 & 7,5 \\
\hline Estudiantes con armas & 89,5 & 5,5 & 5,0 \\
\hline Total & 78,5 & 16,6 & 4,9 \\
\hline Clima escolar & En desacuerdo & De acuerdo & Totalmente de acuerdo \\
\hline Maestros respetuosos & 7,5 & 17,8 & 74,7 \\
\hline Maestros justos & 11,0 & 36,9 & 52,1 \\
\hline Trato justo con quien rompe las reglas & 10,5 & 27,9 & 61,5 \\
\hline Total & 6,7 & 28,9 & 64,4 \\
\hline \multicolumn{4}{|l|}{ Condiciones de inseguridad } \\
\hline Preocupación por violencia & 54,2 & 15,5 & 30,3 \\
\hline Afectado por violencia en comunidad & 76,1 & 14,3 & 9,6 \\
\hline Jóvenes pandilleros & 84,7 & 9,2 & 6,1 \\
\hline Total & 72,1 & 20,5 & 7,4 \\
\hline Bullying & No & Una vez & Dos o más veces \\
\hline Agarrado o empujado & 72,3 & 18,8 & 8,9 \\
\hline Golpeado & 88,0 & 10,3 & 1,7 \\
\hline Le han dañado cosas intencionalmente & 84,6 & 12,8 & 2,6 \\
\hline Amenaza de agresión & 87,9 & 10,0 & 2,1 \\
\hline \multirow[t]{2}{*}{ Humillan } & 73,0 & 19,2 & 7,8 \\
\hline & Nada & Poco & Bastante \\
\hline Le molestan empujones & 76,7 & 18,2 & 5,1 \\
\hline Le molestan golpes & 89,1 & 9,0 & 1,9 \\
\hline Le molesta que dañen & 85,6 & 10,7 & 3,7 \\
\hline Le molestan amenazas & 89,2 & 8,7 & 2,1 \\
\hline \multirow[t]{2}{*}{ Le molesta humillación } & 76,1 & 15,1 & 8,8 \\
\hline & Ausencia & Presencia & \\
\hline Total bullying & 81,7 & 18,3 & \\
\hline
\end{tabular}


agarrados o empujados dos o más veces en la escuela, el $12 \%$ han sido golpeado alguna vez y el $12,1 \%$ recibieron alguna amenaza de agresión (tabla 1).

En la tabla 2, se presenta la evaluación psicométrica de la escala en alusión a la consistencia interna y la fiabilidad de cada uno de sus dominios. En este análisis, se evidenció que los ítems presentan correlaciones superiores a 0,5 con el dominio al que pertenecen, e inferiores a 0,3 con los demás dominios, lo cual corrobora su excelente consistencia interna y poder discriminante. De la misma manera, al evaluar la fiabilidad con el coeficiente alfa de Cronbach, se evidencia que en todos los dominios hay valores superiores a o,8, lo que demuestra que los ítems que componen el instrumento están relacionados, son homogéneos y dan cuenta del clima escolar y la seguridad en la escuela de forma satisfactoria.

Para la validez de contenido, se realizó análisis factorial exploratorio y gráfico de componentes con rotación de varimax en el que se identificaron tres factores que explican el 55,4\% de la varianza de los puntajes de la escala. El primer factor reúne seis items, presenta cargas factoriales entre 0,55 y 0,62, explica el 11,8\% de la varianza y se denominó conductas inseguras; el segundo factor agrupa cuatro ítems con cargas factoriales de 0,48 a 0,71, explica 9,1\% de la varianza y se denominó clima escolar; el tercer factor agrupa diez items con cargas factoriales de o,63 a o,76, explica el $27,5 \%$ de la varianza y se llamó bullying (figura 1).

En la validez de constructo, se comparó el perfil de clima escolar y seguridad en la escuela con el sexo y la zona de residencia, ya que en cada una de estas se presentan características demográficas, económicas y sociales que pueden influenciar la percepción de clima y seguridad en la escuela; en estos análisis, se encontraron diferencias significativas entre los subgrupos (valor $p<0,01$ ), que evidencia que el instrumento discrimina adecuadamente entre grupos teóricamente distintos (tabla 3).

\section{Discusión}

La escala CSCSS presenta excelente fiabilidad y validez en población adolescente de instituciones educativas públicas de Medellín, lo que refleja la disponibilidad de un instrumento corto, de fácil aplicación y con garantías psicométricas en población similar a la estudiada. En alusión a la consistencia interna y la fiabilidad, se demostró que el CSCSS presenta alfa de Cronbach superior a 0,80 en todos los dominios, resultados superiores a la investigación original realizada en 650 estudiantes de California, ya que en ella el alfa de Cronbach para el dominio de conductas inseguras fue de 0,70; clima escolar 0,63; seguridad en la escuela o,61, y victimización 0,75 (Rebelez y Furlong, 2013).

Los estudios realizados en Colombia con instrumentos para evaluar bullying, como la escala de Cisneros (Cepeda-Cuervo, Pacheco-Durán, García-Barco y Piraquive-Peña, 2008) y el cuestionario de intimidación escolar (Moratto, Cárdenas y Berbesí, 2012), presentan buena fiabilidad con alfa de Cronbach de 0,96 y superior a o,80, respectivamente; de la misma manera, la escala para evaluar clima escolar como la escala de clima social escolar (Prado et al., 2010) ha presentado fiabilidad de 0,9; además de estos buenos resultados, el CSCSS presenta la ventaja de evaluar en 15 ítems el clima escolar y el bullying simultáneamente, que facilita la aplicación del instrumento y la interpretación de los resultados.

Con respecto a la validez de contenido, el estudio original identificó tres factores llamados conductas inseguras con cargas factoriales de 0,58 a 0,63; clima escolar con cargas de 0,50 a 0,66; seguridad en la escuela de 0,40 a 0,70; e incluyó una subescala de bullying como un cuarto factor (Rebelez y Furlong, 2013). En contraste, en los adolescentes de Medellín, se formaron tres factores, ya que los ítems que en la escala original constituían el dominio de condiciones de inseguridad se distribuyeron en clima escolar ("La violencia en la escuela es una preocupación para las directivas": lambda de 0,48 ) y conductas inseguras ("la escuela está siendo muy 
ISSN 0123-1294 | e-ISSN 2027-5358 | Educ.Educ. Vol. 20. No. 1 | Enero-abril de 2017 | pp. 9-23.

Universidad de La Sabana | Facultad de Educación

Tabla 2. Análisis de fiabilidad, consistencia interna y poder discriminante

\begin{tabular}{|c|c|c|c|c|}
\hline \multirow{2}{*}{ Conductas inseguras } & \multirow{2}{*}{ Correlación ítem-dominio } & \multicolumn{3}{|c|}{ Correlación ítem-otro dominio } \\
\hline & & Clima escolar & C. inseguridad & Bullying \\
\hline Estudiantes que pelean & $0,594^{* *}$ & $-0,073^{* *}$ & $0,169^{* *}$ & $0,110^{* *}$ \\
\hline Estudiantes que roban & $0,671^{* *}$ & $-0,107^{* *}$ & $0,129^{* *}$ & $0,096^{* *}$ \\
\hline Estudiantes que amenazan & $0,725^{* *}$ & $-0,120^{* *}$ & $0,201^{* *}$ & $0,164^{* *}$ \\
\hline Estudiantes con armas & $0,576^{* *}$ & $-0,095^{* *}$ & $0,202^{* *}$ & $0,131^{* *}$ \\
\hline Éxito (\%) & $100(4 / 4)$ & $100(4 / 4)$ & $100(4 / 4)$ & $100(4 / 4)$ \\
\hline \multicolumn{5}{|c|}{ a de Cronbach: 0,813 } \\
\hline \multicolumn{5}{|c|}{ Varianza explicada: $11,8 \%$} \\
\hline Clima escolar & & $\begin{array}{l}\text { Conductas } \\
\text { inseguras }\end{array}$ & C. inseguridad & Bullying \\
\hline Maestros son respetuosos & $0,728^{* *}$ & $-0,098^{* *}$ & $-0,055^{* *}$ & $-0,103^{* *}$ \\
\hline Maestros son justos & $0,714^{* *}$ & $-0,099^{* *}$ & 0,018 & $-0,072^{* *}$ \\
\hline Trato justo con quien rompe las reglas & $0,681^{* *}$ & $-0,130^{* *}$ & 0,008 & $-0,118^{* *}$ \\
\hline Éxito (\%) & $100(3 / 3)$ & $100(3 / 3)$ & $100(3 / 3)$ & $100(3 / 3)$ \\
\hline \multicolumn{5}{|c|}{ a de Cronbach: 0,838} \\
\hline \multicolumn{5}{|c|}{ Varianza explicada: 9,1\% } \\
\hline C. de inseguridad & & Clima escolar & $\begin{array}{l}\text { Conductas } \\
\text { inseguras }\end{array}$ & Bullying \\
\hline Preocupación por violencia & $0,588^{* *}$ & $0,071^{* *}$ & $0,092^{* *}$ & $0,035^{*}$ \\
\hline Violencia en comunidad & $0,777^{* *}$ & $-0,055^{* *}$ & $0,227^{* *}$ & $0,095^{* *}$ \\
\hline Jóvenes pandilleros & $0,714^{* *}$ & $-0,079^{* *}$ & $0,236^{* *}$ & $0,119^{* *}$ \\
\hline Éxito (\%) & $100(3 / 3)$ & $100(3 / 3)$ & $100(3 / 3)$ & $100(3 / 3)$ \\
\hline \multicolumn{5}{|c|}{ a de Cronbach: 0,830} \\
\hline \multicolumn{5}{|c|}{ Varianza explicada: 7,1\% } \\
\hline Bullying & & C. inseguridad & Clima escolar & $\begin{array}{l}\text { Conductas } \\
\text { inseguras }\end{array}$ \\
\hline Agarrado o empujado & $0,597^{* *}$ & $0,072^{* *}$ & $-0,091^{* *}$ & $0,141^{* *}$ \\
\hline Golpeado & $0,655^{* *}$ & $0,091^{* *}$ & $-0,069^{* *}$ & $0,106^{* *}$ \\
\hline Le han dañado cosas intencionalmente & $0,657^{* *}$ & $0,088^{* *}$ & $-0,118^{* *}$ & $0,134^{* *}$ \\
\hline Amenaza de agresión & $0,645^{* *}$ & $0,113^{* *}$ & $-0,087^{* *}$ & $0,147^{* *}$ \\
\hline Humillan & $0,644^{* *}$ & $0,070^{* *}$ & $-0,089^{* *}$ & $0,124^{* *}$ \\
\hline Le molesta que empujen & $0,640^{* *}$ & $0,072^{* *}$ & $-0,082^{* *}$ & $0,129^{* *}$ \\
\hline Le molestan golpes & $0,635^{* *}$ & $0,076^{* *}$ & $-0,053^{* *}$ & $0,083^{* *}$ \\
\hline Le molesta que dañen & $0,624^{* *}$ & $0,075^{* *}$ & $-0,090^{* *}$ & $0,125^{* *}$ \\
\hline Le molestan amenazas & $0,626^{* *}$ & $0,093^{* *}$ & $-0,077^{* *}$ & $0,114^{* *}$ \\
\hline Le molesta humillación & $0,611^{* *}$ & $0,052^{* *}$ & $-0,070^{* *}$ & $0,088^{* *}$ \\
\hline Éxito (\%) & $100(10 / 10)$ & $100(10 / 10)$ & $100(10 / 10)$ & $100(10 / 10)$ \\
\hline \multicolumn{5}{|c|}{ a de Cronbach: 0,917 } \\
\hline \multicolumn{5}{|c|}{ Varianza explicada: $27,5 \%$} \\
\hline
\end{tabular}

** Se presentaron relaciones significativas en un nivel de o,01. 
Tabla 3. Validez de constructo

\begin{tabular}{|c|c|c|c|c|c|c|c|c|c|c|c|}
\hline & \multicolumn{3}{|c|}{ Conductas inseguras } & \multicolumn{3}{|c|}{ Clima escolar } & \multicolumn{3}{|c|}{ Condiciones inseguridad } & \multicolumn{2}{|c|}{ Victimización } \\
\hline Sexo (\%) & Baja & Moderada & Alta & Malo & Normal & Bueno & Baja & Moderada & Alta & No & Sí \\
\hline Femenino & 82,6 & 13,3 & 4,0 & 6,6 & 27,5 & 65,9 & 73,9 & 19,7 & 6,4 & 82,7 & 17,3 \\
\hline Masculino & 72,2 & 21,6 & 6,2 & 6,8 & 31,1 & 62,1 & 69,5 & 21,6 & 8,9 & 80,3 & 19,7 \\
\hline $\begin{array}{l}\text { Vp chi- } \\
\text { cuadrado }\end{array}$ & \multicolumn{3}{|c|}{ ০,০০০* } & \multicolumn{3}{|c|}{0,060} & \multicolumn{3}{|c|}{$0,005^{*}$} & \multicolumn{2}{|c|}{$0,081 \neq$} \\
\hline \multicolumn{12}{|c|}{ Zona (\%) } \\
\hline Nororiental & 71,9 & 18,3 & 9,8 & 4,9 & 27,7 & 67,4 & 72,8 & 21,0 & 6,3 & 79,0 & 21,0 \\
\hline Noroccidental & 61,9 & 28,7 & 9,4 & 9,8 & 27,2 & 63,1 & 66,9 & 23,9 & 9,1 & 79,3 & 20,7 \\
\hline Centrooriental & 91,4 & 7,0 & 1,6 & 5,5 & 25,7 & 68,8 & 81,1 & 14,5 & 4,4 & 83,5 & 16,5 \\
\hline $\begin{array}{c}\text { Centroocci- } \\
\text { dental }\end{array}$ & 69,9 & 23,9 & 6,2 & 6,5 & 28,3 & 65,2 & 56,2 & 32,2 & 11,6 & 78,6 & 21,4 \\
\hline Suroriental & 82,8 & 14,0 & 3,2 & 5,3 & 35,4 & 59,3 & 67,8 & 22,0 & 10,2 & 87,1 & 12,9 \\
\hline Suroccidental & 83,4 & 14,5 & 2,0 & 5,5 & 30,5 & 64,0 & 79,1 & 16,9 & 4,1 & 84,6 & 15,4 \\
\hline Santa Elena & 70,5 & 23,8 & 5,7 & 5,7 & 23,8 & 70,5 & 66,7 & 27,6 & 5,7 & 82,9 & 17,1 \\
\hline San Cristóbal & 79,9 & 16,1 & 4,0 & 8,5 & 35,3 & 56,3 & 81,7 & 13,8 & 4,5 & 75,9 & 24,1 \\
\hline $\begin{array}{c}\text { San Antonio de } \\
\text { Prado }\end{array}$ & 71,4 & 20,4 & 8,2 & 8,6 & 29,4 & 62,1 & 60,6 & 26,4 & 13,0 & 77,7 & 22,3 \\
\hline $\begin{array}{l}\text { Vp chi- } \\
\text { cuadrado }\end{array}$ & \multicolumn{3}{|c|}{ ০,০০০* } & \multicolumn{3}{|c|}{ O,O०1* } & \multicolumn{3}{|c|}{ O,०००* } & \multicolumn{2}{|c|}{$0,001^{*}$} \\
\hline
\end{tabular}

' Prueba Z*: chi-cuadrado es significativo en el nivel o,o1.

\section{Figura 1. Gráfico de componentes en espacio rotado}

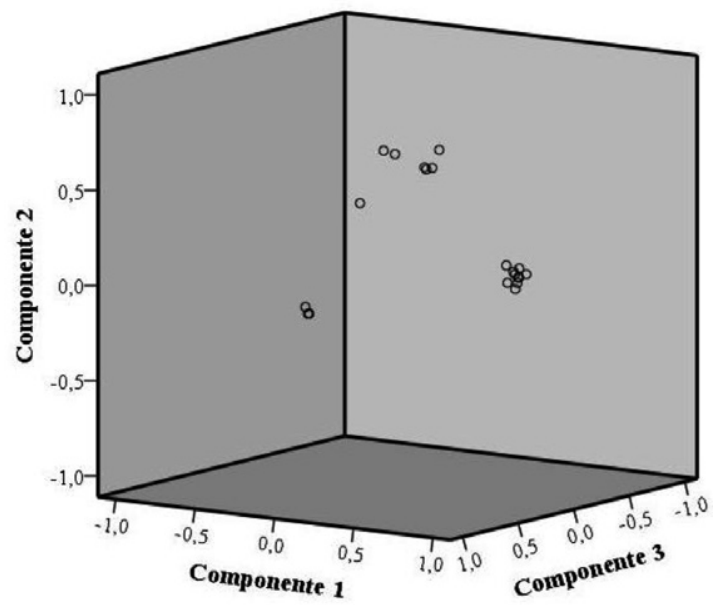

afectada por la violencia en la comunidad": lambda de 0,56 y "La actividad de jóvenes pandilleros está afectando la escuela": lambda de 0,55 ). Es destacable que el dominio de bullying presentó cargas factoriales de 0,63 a o,76, y sus ítems explicaron el 27,5\% de la varianza, es decir, cerca de la mitad de la varianza explicada por el instrumento completo.

La validez de constructo se realizó comparando poblaciones que teóricamente tendrían diferencias en la percepción de clima escolar y bullying, por lo que se tomaron el sexo y las condiciones sociodemógraficas. La comparación por sexo se realizó según estudios previos que utilizan la misma escala y han descrito diferencias por género en todos los dominios del instrumento (Buckley, Storino y Sebastiani, 2003). Esta investigación muestra resultados similares, 
en cuanto los hombres presentaron mayor percepción de conductas inseguras en la escuela ( $V p$ o,ooo) y condiciones de inseguridad más altas (Vp o,005), que evidencia que el instrumento detecta las diferencias por género. De la misma manera, se ha descrito que las formas de manifestación de bullying y percepción del clima escolar difieren por aspectos culturales y socioeconómicos específicos de cada región (Paredes et al., 2011), lo que se corroboró en esta investigación al identificar diferencias significativas entre las zonas en las que se divide la ciudad. Las diferencias que se encontraron por sexo y por zonas de la ciudad afianzan que la escala CSCSS da cuenta del constructo para el cual fue diseñada.

Las propiedades psicométricas de esta escala, en comparación con otras validadas en el país, evidencian algunas limitaciones en las investigaciones locales alrededor del clima escolar y el bullying, ya que, si bien describen buenos niveles de fiabilidad, no reportan propiedades psicométricas como el poder discriminante, la validez de constructo, la validez de contenido. En este sentido, se destacan los esfuerzos por dar cuenta de esta problemática en estudios como el de Chaux et al. (2009), que incluyó 53.316 estudiantes de todo el país; la investigación sobre convivencia escolar de López de Mesa-Melo et al. (2013), en 1091 estudiantes y 101 profesores de instituciones de Cundinamarca; el estudio sobre bullying de Paredes et al. (2008), en 2542 estudiantes de Cali; la investigación de Uribe et al. (2012), en 304 estudiantes de una institución de Santander; y el de Cepeda-Cuervo et al. (2008), en 3226 alumnos de colegios de Bogotá.

Como limitaciones del estudio, se encuentra el sesgo temporal y el no poder realizar una evaluación psicométrica completa donde se incluyeran otras propiedades del instrumento, como la sensibilidad al cambio y la reproducibilidad test-retest. Sumado a lo anterior, debe aclararse que los estudios de evaluación psicométrica generalmente emplean tamaños de muestra menores del actual estudio, ya que las muestras muy grandes pueden inducir el error tipo I o alfa; es decir, que diferencias mínimas en la población sean detectadas como significativas (Edmiston et al., 1993) por el efecto que genera el tamaño de la muestra en la reducción de la amplitud de los intervalos de confianza. Sin embargo, un tamaño de muestra elevado no tiene implicaciones en la fiabilidad, puesto que para el cálculo del alfa de Cronbach es más importante el número de items que hace parte de la escala y sus niveles que el número de participantes (Campo-Arias y Oviedo, 2008). Esta misma consideración, se tiene para el análisis de consistencia interna y poder discriminante vía correlaciones de Spearman, dado que en estos parámetros se destaca el valor de los coeficientes de correlación más que la significación estadística (Pita y Pértega, 1997).

Por otro lado, cabe la posibilidad de que en la validez de constructo se haya incurrido en error tipo alfa; sin embargo, el error estándar para las proporciones de las subescalas estuvo entre 0,5 y 0,9; de modo que la probabilidad del error tipo I resulta más baja que las diferencias halladas en la proporción de conductas inseguras, clima escolar, seguridad en la escuela y bullying entre hombres y mujeres, y en las siete zonas estudiadas, lo que confirma que las diferencias reportadas no son un artefacto estadístico, sino que son significativas a la luz del fenómeno estudiado.

En cuanto a la complementación del análisis factorial exploratorio con un método confirmatorio y con una muestra nueva, existen autores que indican que estos análisis podrían ser redundantes; por ello, este tema es aún controversial (Pérez-Gil, Chacón y Moreno, 2000). Asimismo, en los estudios de validación de escalas la correlación de Pearson es la prueba más común para evaluar la consistencia interna; no obstante, esta prueba se restringe a variables continuas, de manera que en esta investigación se utilizó la correlación de Spearman que equivale a la correlación de Pearson, con la diferencia de que admite variables ordinales (Pita Fernández y Pértega Díaz, 1997). 
Pese a las limitaciones descritas, se demostró que la escala CSCSS es un cuestionario corto, de rápida administración, fiable y válido, que puede ser utilizado en población adolescente de Medellín para monitorear clima escolar y bullying, evaluar programas de intervención, establecer un sistema de evaluación periódica e identificar niveles de alerta para orientar acciones de prevención. Las futuras investigaciones deberían evaluar el desempeño de la escala en otras regiones del país y en estudiantes de educación primaria, con el fin de poner en evidencia la disponibilidad de un instrumento que permita establecer un sistema de vigilancia nacional sobre el clima escolar y el bullying.

\section{Referencias}

Analitis, F., Velderman, M. K., Ravens-Sieberer, U., Detmar, S., Erhart, M., Herdman, M. et al. (2009). Being bullied: Associated factors in children and adolescents 8 to 18 years old in 11 European countries. Pediatrics, 123(2), 569-577. doi:10.1542/peds.2008-0323.

Buckley, M., Storino, M. y Sebastiani, A. (2003). The impact of school climate: Variation by ethnicity and gender. Poster session presented at the 111th Annual Meeting of the American Psy chological, Toronto.

Campo-Arias, A. y Oviedo, H. (2008). Propiedades psicométricas de una escala: la consistencia interna. Revista de salud pública, 10 (5), 831-839.

Cassiani-Miranda, C. A., Gómez-Alhach, J., Cubides-Munévar, A. M. y Hernández-Carrillo, M. (2014). Prevalencia de bullying y factores relacionados en estudiantes de bachillerato de una institución educativa de Cali, Colombia, 2011. Revista de salud pública, 16(1), 14-26.

Cepeda-Cuervo, E., Pacheco-Durán, P. N., García-Barco, L. y Piraquive-Peña, C. (2008). Acoso escolar a estudiantes de educación básica y media. Revista de salud pública, 10 (4), 517-528.

Chaux, E., Molano, A. y Podlesky, P. (2009). Socio-economic, socio-political and socio-emotional variables explaining school bullying: A country-wide multilevel analysis. Aggressive Behavior, 35, 520-529. doi: 10.1002/ ab.2O32O.

D’Angelo, L.y Fernández L. (2010). Clima, conflictos y violencia en la escuela: un estudio en escuelas secundarias de gestión pública y privada del Área Metropolitana de Buenos Aires. Buenos Aires: Unicef-Flacso

Edmiston, C. E., Josephson, A., Ottinger J., Ciacco-Tsivitis, M. y Palenik, C. (1993). The numbers game: Sample-size determination. American Journal of Infection Control, 21(3), 151-154.

Eljach, S. (2011). Violencia escolar en América Latina y el Caribe Superficie y fondo. Panamá: Unicef.

Fidalgo, A. M. y Piñuel, I. (2004). La escala Cisneros como herramienta de valoración del mobbing. Psicothema, $16(4), 615-624$.

Fischer, R. M. (2010). Bullying escolar no Brasil: Relatório final. São Paulo: Centro de Empreendedorismo Social e Administração em Terceiro Setor, Fundação Instituto de Administração. 
ISSN 0123-1294 | e-ISSN 2027-5358 | Educ.Educ. Vol. 20. No.1 | Enero-abril de 2017 | pp. 9-23.

Universidad de La Sabana | Facultad de Educación

Flores, P. K. (2009). Por el derecho a una vida escolar sin violencia. La Paz: Asociación Voces Vitales.

Heinsohn, R., Chaux, E. y Molano, A. (2010). “La chispita que quería encender todos los fósforos”: percepciones, creencias y emociones frente a la intimidación en un colegio masculino. Voces y Silencios: Revista Latinoamericana de Educación, 1(1), 5-22.

Infante, L., Hierrezuelo, L., García, B., Sánchez, A., De La Morena, M. L., Muñoz, A. y Trianes M. V. (2003). Evaluación de actitudes violentas y clima escolar en situaciones de agresividad en alumnado de secundaria. Psicología, Saúde y Doencas, 4(2), 277-286.

López de Mesa-Melo, C. M., Carvajal-Castillo, C. A., Soto-Godoy, M. F.y Urrea-Roa, P. N. (2013). Factores asociados a la convivencia escolar en adolescentes. Educación y Educadores, 16(3), 383-410.

López, V., Bilbao, M. y Rodríguez, J. (2012). La sala de clases sí importa: incidencia del clima de aula sobre la percepción de intimidación y victimización entre escolares. Universitas Psychological, 11(1), 91-101.

Moratto, N., Cárdenas, N. y Berbesí, D. (2012). Validación de un cuestionario breve para detectar intimidación escolar. Revista CES Psicología, 5(2), 70-78.

Nansel, T. R., Overpeck, M., Pilla, R. S., Ruan, W. J., Simons-Morton, B.y Scheidt, P. (2001). Bullying behaviors among US youth: Prevalence and association with psy chosocial adjustment. JAMA, 285(16), 2094-2100.

Ortega, R., Elipe, P., Mora-Merchán, J., Genta, M. L., Brighi, A., Guarini, A. et al. (2012). The emotional impact of bullying and cyberbullying on victims: A european cross-national study. Aggressive Behavior, 38(5), 342-356. doi: 10.1002/ab.21440

Orue, I. y Calvete, E. (2010). Elaboración y validación de un cuestionario para medir la exposición a la violencia en infancia y adolescencia. International Journal of Psychology and Psychological Therapy, 10(2), 279-292.

Paredes, M. T., Álvarez, M. C., Lega, L. I. y Vernon, A. (2008). Estudio exploratorio sobre el fenómeno del "bullying" en la ciudad de Cali, Colombia. Revista Latino Americana de Ciencias Sociales en Niñez y Juventud, 6(1), 295-317.

Paredes, M. T., Lega, L. I., Cabezas, H., Ortega, M. E., Medina, Y. y Vega, C. (2011). Diferencias transculturales en la manifestación del bullying en estudiantes de escuela secundaria. Revista Latinoamericana de Ciencias Sociales, Niñez y Juventud, 2(9), 761-768.

Pérez-Gil, J. A., Chacón, M. S. y Moreno, R. (2000). Validez de constructo: el uso de análisis factorial exploratorioconfirmatorio para obtener evidencias de validez. Psicothema, 12(2), 442-446.

Personería de Medellín (2013). Informe sobre la situación de los derechos humanos en la ciudad de Medellín. Recuperado de http://www.personeriamedellin.gov.co/

Pita Fernández, S. y Pértega Díaz, S. (1997). Relación entre variables cuantitativas. Unidad de Epidemiología Clínica y Bioestadística. Complexo Hospitalario Juan Canalejo. Cadernos de Atención Primaria, 4, 141-144. 
Prado D.V., Ramírez, M. M. y Ortiz C. M. (2010). Adaptación y validación de la escala de clima escolar (CES). Revista Electrónica Actualidades Investigativas en Educación, 10(2), 1-13.

Rebelez, J. L. y Furlong, M. J. (2013). Psy chometric support for an abbreviated version of the California school climate and safety survey. International Journal of School \& Educational Psychology, 1(3), 154-165. doi: $10.1080 / 21683603.2013 .819306$

Rodríguez-Jiménez, O. y Martínez-Rojas, J. G. (2009). Cinco instrumentos objetivos para medir el maltrato por abuso de poder entre pares. Avances en medición, 7, 129-134.

Sánchez, S., Pizza, L.y Rojas, L. (2009). Evaluación de actitudes violentas y clima escolar en situaciones de agresividad en alumnos de sexto grado del IED Gabriel Betancourt Mejía. Perspectivas contemporáneas en psicología social. Bogotá: Universidad Nacional de Colombia.

Síndic de Greuges de Catalunya (2006). Convivencia y conflictos en los centros educativos. Barcelona: Sindic de Greuges de Catalunya.

Torregrosa, M. S., Inglés, C. J., Estévez-López, E., Musitu, G. y García-Fernández, J. M. (2011). Evaluación de la conducta violenta en la adolescencia: revisión de cuestionarios, inventarios y escalas en población española. Aula Abierta, 39(1), 37-50.

Turkemen, N., Dokgoz H., Akgoz, S., Eren B., Vural, P.y Polat, O. (2013). Bullying among high school students Turkey. Maedica a Journal of Clinical Medicine, 8(2), 143-152.

Uribe, A. F., Orcasita, L. T. y Gómez, E. A. (2012). Bullying, redes de apoyo social y funcionamiento familiar en adolescentes de una institución educativa de Santander, Colombia. Psychologia. Avances de la disciplina, 6(2), 83-99. 
